\title{
Training-related changes in the R-R interval at the onset of passive movements in humans
}

\author{
L.C. Vianna1 ${ }^{1}$ D.R. Ricardo ${ }^{2}$ and C.G.S. Araújo ${ }^{1,3}$ \\ ${ }^{1}$ Programa de Pós-Graduação em Educação Física, Universidade Gama Filho, Rio de Janeiro, RJ, Brasil \\ ${ }^{2}$ Faculdade de Ciências Médicas e da Saúde de Juiz de Fora, SUPREMA, Juiz de Fora, MG, Brasil \\ ${ }^{3}$ Clínica de Medicina do Exercício (CLINIMEX), Rio de Janeiro, RJ, Brasil
}

Correspondence to: C.G.S. Araújo, Clínica de Medicina do Exercício (CLINIMEX), Rua Siqueira Campos, 93/101, 22031-070 Rio de Janeiro, RJ, Brasil

Fax: +55-21-2549-4295. E-mail: cgaraujo@iis.com.br

\begin{abstract}
The aim of the present study was to determine whether training-related alterations in muscle mechanoreflex activation affect cardiac vagal withdrawal at the onset of exercise. Eighteen male volunteers divided into 9 controls (26 \pm 1.9 years) and 9 racket players ( $25 \pm 1.9$ years) performed $10 \mathrm{~s}$ of voluntary and passive movement characterized by the wrist flexion of their dominant and non-dominant limbs. The respiratory cycle was divided into four phases and the phase $4 \mathrm{R}-\mathrm{R}$ interval was measured before and immediately following the initiation of either voluntary or passive movement. At the onset of voluntary exercise, the decrease in R-R interval was similar between dominant and non-dominant forearms in both controls (166 \pm 20 vs $180 \pm 34$ ms, respectively; $P>0.05)$ and racket players (202 \pm 29 vs $201 \pm 31 \mathrm{~ms}$, respectively; $P>0.05)$. Following passive movement, the non-dominant forearm of racket players elicited greater changes than the dominant forearm (129 $\pm 30 \mathrm{vs} 77 \pm 17 \mathrm{~ms} ; \mathrm{P}<0.05)$, as well as both the dominant $(54 \pm 20 \mathrm{~ms} ; \mathrm{P}<0.05)$ and non-dominant $(59 \pm 14 \mathrm{~ms} ; \mathrm{P}<0.05)$ forearms of control subjects. In contrast, changes in R-R interval elicited by the racket players' dominant forearm were similar to that observed in the control group, indicating that changes in R-R interval at the onset of passive exercise were not attenuated in the dominant forearm of racket players. In summary, cardiac vagal withdrawal induced by muscle mechanoreflex stimulation is well-maintained, despite long-term exposure to training.
\end{abstract}

Key words: Mechanoreceptors; Vagus nerve; Muscle stretching exercises; R-R interval

L.C. Vianna was supported by a scholarship from CNPq (\#142017/2006-0), and C.G.S. Araújo is the recipient of a CNPq scientific scholarship (\#301832/2005-6).

Received September 27, 2007. Accepted August 18, 2008

\section{Introduction}

At the onset of exercise, there is an immediate and rapid decrease in $R-R$ interval mediated purely by the abrupt withdrawal of cardiac vagal activity (1-3). This instantaneous decrease in cardiac vagal activity is induced by central signals from the higher brain (central command) and by neural feedback arising from mechanically sensitive skeletal muscle receptors (the muscle mechanoreflex) (4-6). Moreover, al-Ani et al. (7) demonstrated that the initial changes in R-R interval in response to muscle con- traction are greater after training than before training. However, the underlying neural cardiovascular mechanisms of training-related alterations in R-R interval responses at the onset of exercise are not understood. Thus, previous studies have addressed this issue considering the possibility of neural changes in the relative role of the muscle mechanoreflex and its inhibitory effect on cardiac vagal activity (8-12). Additionally, passive movement is widely employed to estimate the effect of the muscle mechanoreflex in humans $(10,13-15)$, because the effect of central command can be removed. 
Human studies examining the influence of exercise training on the heart rate response at the onset of passive movement have used a cross-sectional design $(11,12)$, making comparisons between trained and untrained subjects. These investigators noted that the heart rate increase throughout $20 \mathrm{~s}$ of passive movement is attenuated in endurance runners (11) and sprinters (12) compared to untrained subjects. Of note, a potential problem in trying to evaluate the relative importance of these mechanoreflex adaptations has been the inability to completely dissociate them from central adaptations. Nevertheless, the heart rate response at the onset of passive movement seems to be similar for dominant and non-dominant limbs (10), although the dominant limb may be more trained compared with the non-dominant limb as a result of daily activity. However, the low-level daily activity may possibly mask a training-induced peripheral neural adaptation in dominant limbs. Taken together, these authors suggested that the decreased response in heart rate may be due to desensitization of muscle mechanoreceptors. Although this suggests training-related changes in muscle mechanoreflex, a potential caveat is that these studies did not carefully isolate the muscle mechanoreflex component of the neural feedback from the working muscle, since 20 s of exercise is enough time to activate chemically sensitive skeletal muscle receptors (the muscle metaboreflex) $(5,16)$. In addition, these studies did not aim to isolate the vagally mediated $R-R$ interval response, since the R-R interval is also modulated by sympathetic activity in exercise of this duration $(2,17,18)$.

On the basis of these considerations, it is currently unknown whether these training-related alterations in muscle mechanoreflex activation are apparent in the cardiac vagal withdrawal at the onset of exercise. Therefore, the present study was designed to investigate R-R interval changes at the onset of passive movement of dominant and non-dominant limbs in racket players and control subjects. In addition, in order to test the inhibitory influence of muscle mechanoreflex stimulation solely on cardiac vagal control, we assessed only the R-R interval immediately following passive movement over one respiratory cycle $(7,19,20)$. We hypothesized that the R-R interval response at the onset of passive movement would be attenuated in the dominant limb in racket sport players, suggesting a decrease in muscle mechanoreflex sensitivity.

\section{Subjects and Methods}

\section{Subjects}

Nine normal volunteers and 9 racket players $(8$ tennis and 1 squash players) participated in this study. The racket players recruited in the present study reported playing for at least 8 years, currently engaging in $15 \mathrm{~h}$ of play per week. All control subjects had previously been involved in various sports, although none consisting of racket sports or unilateral activities. All subjects were right-handed and, according to a previous study (21), we defined the upper limb utilized to play racket sports as dominant. All subjects were non-smokers, non-obese, normotensive and asymptomatic for cardiovascular or respiratory disease and none of them were taking any medication.

The study was approved by the University Ethics Committee and conformed to the Declaration of Helsinki. All subjects gave written informed consent to participate.

\section{Experimental protocol}

Subjects were asked to refrain from consumption of caffeinated beverages and exercise for at least $12 \mathrm{~h}$ before the tests and not to eat in the $2 \mathrm{~h}$ preceding the tests. All measurements were made in a room free from external distractions in which only the researcher or a nurse assistant and the subject were present. Prior to the experimental measurements, subjects were habituated with all apparatuses and protocols. In one visit, subjects performed 4 trials, assigned in a counterbalanced order and separated by at least $5 \mathrm{~min}$, as follows: 1) Dominant voluntary exercise; 2) Non-dominant voluntary exercise; 3) Dominant passive movement; 4) Non-dominant passive movement. The protocol included 2 min of rest followed by $10 \mathrm{~s}$ of voluntary exercise or passive movement, characterized by wrist flexion at $2 \mathrm{~Hz}$ following an audio signal. Subjects were seated comfortably throughout the experiments with their backs against an experimental chair and their forearms supported on a table in front of them. During the brief voluntary exercise, all subjects were instructed to hold a dumbbell with an equivalent weight of $\sim 4 \%$ of the subject's body mass in their hand, with the palm facing upward. The start of voluntary exercise was signaled by the experimenter before the start of an inspiratory period. The onset point, just before the start of the inspiratory period, was determined by the respiratory movement curve monitored on the oscilloscope. Passive movement was carried out by the experimenter manually flexing the subject's wrist to the end of the comfortable range of motion without the dumbbell. All subjects were asked to relax and not to resist the movement, with care being taken to keep the body as stable as possible in order to avoid motion artifacts. To ascertain whether muscles actually contracted during passive movement, bipolar electrodes were attached to the wrist flexors to detect any electromyographic signals produced. Respiratory rate was controlled by asking the subject to breathe in time to a metronome set to maintain a 
respiratory rate that was comfortable for each individual. This respiratory frequency was kept constant throughout the experimental protocols.

\section{Measured variables}

The electrocardiogram (ECG) was obtained by one lead with a standard CM5 configuration, comprising three silver chloride monitoring electrodes placed on the chest. The ECG was recorded at a sampling frequency of 1000 $\mathrm{Hz}$ (Powerlab 4/25T and software Chart 5, AD Instruments, Australia). The respiratory movements and the phase of the respiratory cycle were measured using a pneumotachograph (MLT1000L, AD Instruments). The electromyogram (EMG) was recorded from the wrist flexor muscles throughout the experimental protocols and was detected using bipolar golden surface electrodes. Prior to application of the surface electrodes, the site was prepared by removal of dead skin by abrasion and cleaning with alcohol. Conducting gel was applied to the electrodes before they were placed on the central portion of the muscle belly in a direction parallel to muscle fiber course. Movement artifacts were minimized by taping the electrodes and wires to the skin. For analysis, EMG signals were rectified and sampled at a frequency of $1000 \mathrm{~Hz}$ using a bandpass filter of 30 to $500 \mathrm{~Hz}$. Maximal handgrip strength (22) of dominant and non-dominant limbs was determined as the higher value of two trials performed before the experiment in the standing position with a specific dynamometer (Grip Dynamometer, Takei Kiki Kogyo, Japan). Forearm girths were measured at the largest position (23).

\section{Data analysis}

For analysis purposes, the respiratory cycle was divided into four phases (1 to 4 ) of equal duration from the beginning of inspiration to the end of expiration. Since the R-R interval varies throughout the respiratory cycle, $R-R$ interval values were measured at identified phases of the cycle. Phases 1 and 2 relate to early and late inspiration and phases 3 and 4 relate to early and late expiration, respectively. For each trial, mean values were calculated for the phase-4 R-R interval for $30 \mathrm{~s}$ before each trial and the first phase-4 R-R interval immediately following the initiation of either voluntary or passive movement $(7,19,20)$. The difference in these two R-R interval measurements was then calculated for each trial to obtain the change in R-R interval upon voluntary or passive movement expressed in milliseconds and as a percentage. Phase 4 in the respiratory cycle was chosen because the cardiac vagal activity is greatest at this time (20), expressed by a longer R-R interval duration. The morphological impact of the racket sport training was calculated by the effect size (24), using the differences in handgrip strength and forearm girth between dominant and nondominant limbs. Various methods have been described to estimate the magnitude of an effect size and Cohen's $d$ has been shown to be the most common and appropriate method. Cohen's $d$ represents the difference between two means, divided by the variability amongst the sample. Also, forearm training stimulus was calculated by the relative side-to-side difference $=($ dominant - non-dominant $) /$ non-dominant $)^{\star} 100$.

\section{Statistical analysis}

Data are reported as mean \pm SEM. Statistical comparisons of physiological variables were made by three-way analysis of variance (ANOVA) for repeated measures, in which group (controls and players), dominance (dominant and non-dominant limbs) and exercise paradigm (voluntary and passive) were the main factors, followed by the Fisher least significant difference test for post hoc pairwise comparisons. The results for control and player subjects concerning physical characteristics were compared by the paired and unpaired Student $t$-tests. Adequate sample size was calculated for ANOVA with 4 groups (within factors), applying a desired power of 0.80 and an $\alpha$ error of $5 \%$. Using the effect size of 0.75 , the minimum sample size was determined to be 9 subjects in each group (i.e., control + players $=18$ subjects). Statistical significance was set at $P \leq 0.05$. All analyses were conducted using the Statistical Package for the Social Sciences, version 13 (SPSS, USA).

\section{Results}

\section{Subject characteristics}

The physical characteristics of the subjects are summarized in Table 1. The dominant forearms of the control group had a greater girth than the non-dominant forearms $(P<0.05)$. This represents only a $0.3-\mathrm{cm}$ difference between the two forearms. However, in the racket player group, the difference in forearm girth was significantly greater $(P<0.05)$, corresponding to a $1.8-\mathrm{cm}$ difference in girth between dominant and non-dominant forearms. As expected, the relative side-to-side difference in the racket player group was greater than in the control group (Table 1). Maximal handgrip strength was greater in the dominant forearms of both the control and racket player groups. However, both groups had dissimilar relative side-to-side difference between dominant and non-dominant forearms $(P<0.05)$. In this respect, the effect size clearly shows the influence of racket sport training on the differences between dominant and non-dominant forearms.

\section{$\mathbf{R}-\mathbf{R}$ interval responses at the onset of exercise}

At rest, there were no significant differences in phase- 
$4 \mathrm{R}-\mathrm{R}$ interval within trials $(\mathrm{P}>0.05$; Table 2$)$. Figure 1 shows the $\mathrm{R}-\mathrm{R}$ interval changes ( $\Delta$ values) during voluntary and passive movements in both the dominant and non-dominant forearms of controls and racket players. In all trials, the R-R interval was significantly decreased, i.e., heart rate increased with exercise $(P<0.05$; Figure 1$)$. This decrease in the R-R interval was similar between dominant and non-dominant forearms in both controls (18 \pm 2.2 vs 19 $\pm 3.4 \%$, respectively; $\mathrm{P}>0.05)$ and racket players $(22 \pm 3.1$ vs $21 \pm 3.0 \%$, respectively; $\mathrm{P}>0.05$ ) at the onset of voluntary exercise. Also, no differences were found in this response between groups. Following passive movement, the non-dominant forearm of racket players elicited a significantly larger change in $\mathrm{R}-\mathrm{R}$ interval than the dominant forearm $(13 \pm 2.5$ vs $8 \pm 1.5 \%$; $P<0.05)$. In addition, this response was larger than that elicited by both dominant $(6 \pm$ $2.2 \% ; \mathrm{P}<0.05)$ and non-dominant $(6 \pm 1.5 \% ; \mathrm{P}<0.05)$ forearms in controls during passive movements. Unexpectedly, the R-R interval change elicited by the dominant forearm of the racket players was similar to that observed in

Table 1. Characteristics of the subjects studied.

\begin{tabular}{lcc}
\hline & $\begin{array}{c}\text { Racket players } \\
(\mathrm{N}=9)\end{array}$ & $\begin{array}{c}\text { Control subjects } \\
(\mathrm{N}=9)\end{array}$ \\
\hline Age (years) & $25 \pm 1.9$ & $26 \pm 1.9$ \\
Height (cm) & $180.2 \pm 2.2$ & $179.8 \pm 1.8$ \\
Weight (kg) & $77.2 \pm 3.3$ & $78.9 \pm 1.5$ \\
Forearm girth (cm) & & \\
$\quad$ Dominant arm & $28.5 \pm 0.4$ & $28.1 \pm 0.5$ \\
Non-dominant arm & $26.7 \pm 0.3^{*}$ & $27.8 \pm 0.5^{*}$ \\
Side-to-side difference (\%) & $6.7 \pm 0.6$ & $1.1 \pm 0.3^{+}$ \\
Effect size & 1.38 & 0.20 \\
Handgrip strength (kg) & & \\
$\quad$ Dominant arm & $53.4 \pm 2.1$ & $50.1 \pm 1.6$ \\
$\quad$ Non-dominant arm & $45.7 \pm 1.6^{*}$ & $47.1 \pm 1.6^{*}$ \\
Side-to-side difference (\%) & $17.4 \pm 3.5$ & $9.1 \pm 1.6^{+}$ \\
Effect size & 1.63 & 0.61 \\
\hline
\end{tabular}

Data are reported as means \pm SEM. The effect size quantified the difference between two groups.

${ }^{*} \mathrm{P}<0.05$ compared to the dominant arm (paired Student $t$-test); ${ }^{+} \mathrm{P}<0.05$ compared to racket players (unpaired Student $t$-test).

Table 2. Baseline values of phase-4 R-R interval.

\begin{tabular}{lccccc}
\hline & \multicolumn{2}{c}{ Voluntary } & & \multicolumn{2}{c}{ Passive } \\
\cline { 2 - 3 } \cline { 5 - 6 } & Dominant & Non-dominant & & Dominant & Non-dominant \\
\hline Control & $948 \pm 28$ & $937 \pm 23$ & & $943 \pm 16$ & $977 \pm 22$ \\
Racket players & $910 \pm 51$ & $918 \pm 45$ & & $958 \pm 60$ & $956 \pm 69$ \\
\hline
\end{tabular}

Data are reported as means \pm SEM in $\mathrm{ms}$. controls in both dominant and non-dominant forearms. These results were also observed when absolute changes of the $\mathrm{R}$ $R$ interval were calculated from rest values (Figure 1).

\section{Discussion}

In this study, we attempted to elucidate whether training-related changes in muscle mechanoreflex sensitivity are apparent in the cardiac vagal withdrawal at the onset of passive movement. To our knowledge, this is the first study to use the approach of passive movement in racket players to test the hypothesis that long-term training is critical to the sensitization of muscle mechanoreceptor control of cardiac vagal withdrawal at the onset of exercise. This was accomplished by assessing R-R interval changes in response to forearm muscle stimulation via passive movement of the wrist performed for $10 \mathrm{~s}$ because only the R-R interval changes in the first respiratory cycle after exercise onset were considered for the present study. The major new finding of the present study is that, despite greater decreases in R-R interval at the onset of passive movement in the non-dominant forearm in racket players, no differences in R-R interval were observed between the dominant forearm of racket players and controls. Of note, similar results were obtained in both absolute and relative analyses of $\mathrm{R}-\mathrm{R}$ interval changes. We had hypothesized that the R-R interval response at the onset of passive movement would be attenuated in the dominant limb of racket players, suggesting a decrease in muscle mechanoreflex sensitivity due to cumulative training exposure. However, despite greater differences in girths and handgrip strength between dominant and non-dominant forearms in racket players, the present result clearly negates this hypothesis.

Training studies have provided some insight into the mechanisms that might be related to a reduced chronotropic response due to an attenuated muscle mechanoreflex $(11,12,25,26)$, although our results clearly reject this hypothesis. Fisher and White (27) suggested that in muscles that are regularly exposed to the products of anaerobic exercise, such as the forearm muscles of racket players, the muscle mechanoreflex may become desensitized. In other words, chronic exposure to muscle acidosis induced by racket sport training could attenuate the muscle mechanoreflex-induced changes in R-R interval in response to a dominant forearm exercise. Sinoway et al. (26) reported that the repeated infusion of 
lactic acid into cats leads to a progressive decline in the discharge frequency of mechanically sensitive group III muscle afferents. In addition, two previous studies by a Japanese research group $(11,12)$ showed attenuated heart rate responses at the onset of dynamic exercise in endurance runners and sprinters compared to untrained subjects. In contrast, although sprint training involves both high-intensity interval and strength training, Carrington et al. (8) did not observe differences in chronotropic response between sprinters and untrained subjects during 2 min of involuntary isometric muscle contraction. Given a possible greater disturbance in muscle mechanoreflex sensitivity in trained muscle, we supposed that cardiac vagal withdrawal (i.e., R-R interval changes) would be attenuated in the dominant forearm in racket sport players at the onset of passive exercise, suggesting a decrease in muscle mechanoreflex sensitivity. This idea was not supported by the present finding.

The other important but unexpected finding of the present study was the marked change in R-R interval noted exclusively in the non-dominant forearm of the racket player during passive movement. This response was significantly greater than in the dominant forearm of racket players or in either forearm of controls. However, it is difficult to determine which mechanism is responsible for this response. One reasonable possibility is that muscle mechanoreflex sensitivity is increased in the non-dominant forearm of racket players. In agreement with previous studies $(25,28,29)$, the forearm girth and handgrip strength were greatest in the dominant forearm of racket players, followed by the dominant forearm of controls, the nondominant forearm of controls, and finally the non-dominant forearm of racket players, which seemed to be "the weakest of all". One explanation for this may be that among all four forearms, the racket players' non-dominant limb was the least used, and thus the weakest, as can be seen from the mean values of maximal handgrip strength (Table 1). Interestingly, this phenomenon was seen only in "young starters" $(28,29)$. It is thus possible that during the rapid phase of growth, the less used non-dominant limb of the racket players is left somewhat behind developmentally. Given this idea, this explanation could be related to the interesting finding that muscle mechanoreceptor sensitivity is increased in heart failure patients (13) who are restricted in daily life activities compared with normal subjects. In addition, Hayashi et al. (30) provided the first demonstration of the effects of disuse atrophy on the muscle mechanoreflex in animal models. These investigators demonstrated that the atrophied muscle increases the pressor response to muscle stretch in rats, due to sensitization of the muscle mechanoreflex (30). However, the mechanisms underlying changes in muscle mechanoreflex sensitivity are still unclear. It is currently unknown whether these muscle mechanoreflex adaptations are apparent in the non-dominant forearm of racket players and we agree that further studies are needed to address this issue in relation to our results.

Another finding of our investigation is that during voluntary exercise there were no differences in $R-R$ interval changes between dominant and non-dominant limbs within and between groups. Previous studies $(8,12,21)$ have reported a similarly chronotropic response between trained and untrained subjects during voluntary exercise. This could be explained by the redundancy in the cardiovascular control system $(14,31,32)$, which may allow central command to mask any changes in muscle mechanoreflex sensitivity in the target muscle. During passive movement, the influence of central command on the R-R changes is

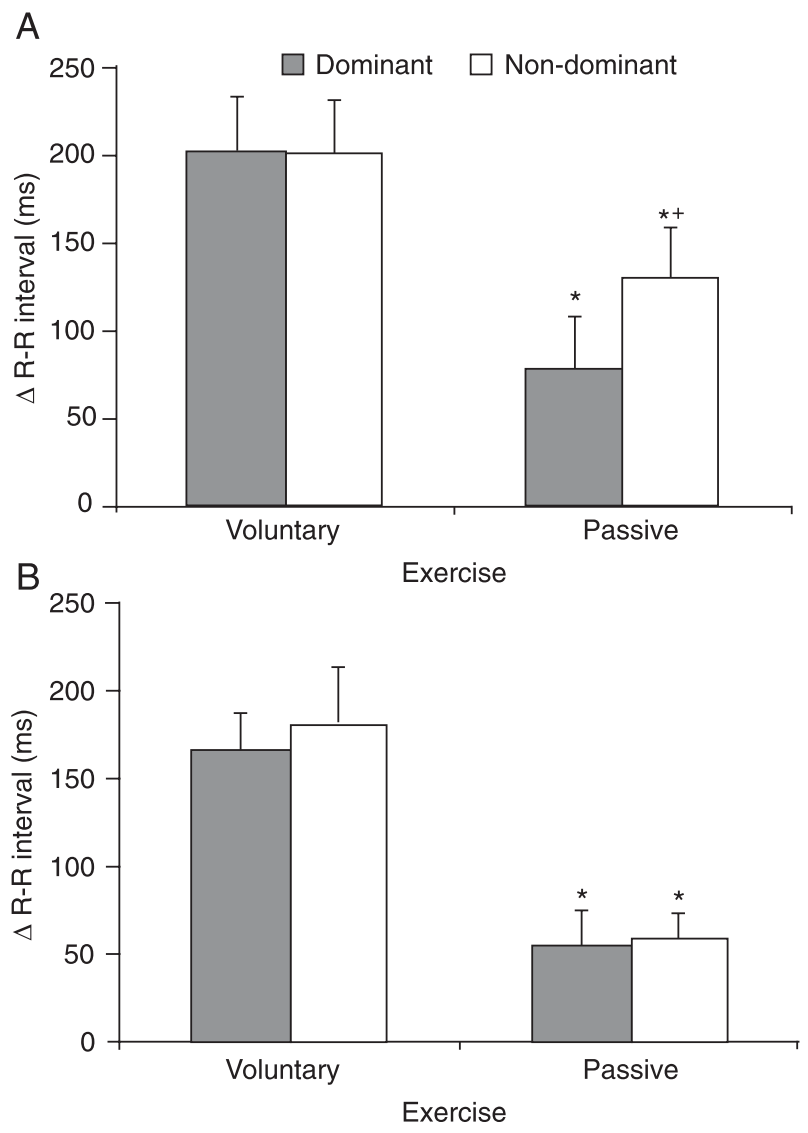

Figure 1. Absolute changes from rest of R-R interval during voluntary and passive movement in racket players $(A)$ and control subjects (B). Filled columns: dominant; open columns: nondominant. ${ }^{*} \mathrm{P} \leq 0.05$ compared to voluntary exercise; ${ }^{+} \mathrm{P} \leq 0.05$ compared to racket players' dominant limb and both forearms of control subjects (Fisher least significant difference test for post hoc pairwise comparisons). 
removed and a clearer picture of the role of the muscle mechanoreflex input to the heart is revealed. In the present study, although R-R interval responsiveness differed between dominant and non-dominant forearms in racket players during passive exercise, $R-R$ interval changes were equally maintained in both the dominant and nondominant limbs in control subjects. Hotta et al. (10) have also reported a similar heart rate response between dominant and non-dominant limbs during either voluntary or passive movement in non-racket players, although these investigators expected an attenuated peripheral neural reflex to the heart.

Previous studies have also used racket players as a model to investigate peripheral adaptations to training. Sinoway et al. (33) reported that the reactive hyperemic blood flow coupled with 1 min of exercise was $42 \%$ higher in the dominant than the non-dominant forearm of racket players. Saito et al. (21) determined whether exerciseinduced muscle sympathetic nerve activation might be altered by training, by comparing dominant and non-dominant forearms of tennis players. In addition, the advantage of this model (i.e., comparing dominant and non-dominant forearms within subjects) was to exclude the effect of interindividual differences related to central adaptations to training, especially under resting conditions, which was not the case in previous studies $(8,11,12)$. In the present study, the similar resting conditions between trials was important for our hypothesis, since R-R interval responses at the onset seem to be dependent upon the level of cardiac vagal activity $(1,34)$. Studies in animals $(1)$ and in humans (34) have reported that the $R-R$ interval responses during the first seconds of exercise are directly associated with vagal activity (i.e., when vagal activity is high, the magnitude of the response is increased). However, this concern might not apply to the present study, which was carried out with "trained" and "untrained" skeletal muscle in the same subjects, thus circumventing this potential problem.

\section{Limitations \\ Several limitations of the design and interpretation of the present investigation should be considered. First, al- though dynamic passive movement is widely employed to isolate the muscle mechanoreflex from central command and muscle metaboreflex activation in humans, it was not possible to quantify the amount of muscle mechanoreflex stimulation generated in the target muscle throughout the}

passive movement, or mimic the exact patterns of motor activation evoked during voluntary exercise. Indeed, this is the potential weakness of this model, even though it has been used by our research group $(14,15)$ and others $(13,35)$. In addition, it is probable that our results were not representative of sustained muscle stretch (36), muscle compression (37) or electrical stimulation (9). Second, the present results were unlikely to be influenced by muscle metaboreflex and/or sympathetic outflow because only the $R-R$ interval changes in the first respiratory cycle after exercise onset $(<5 \mathrm{~s}$ ) were considered. Both build-up of metabolites within the working muscle (16) and increases in cardiac sympathetic activity $(7,20)$ may require several seconds (>5 s) and would not influence $R-R$ interval changes during the very short duration of the exercise performed in the present study. Third, another important physiological concern is that heart rate may be selectively modulated by hemisphere laterality. Saito (38) demonstrated that heart rate during the first minute of static handgrip exercise was lower in the right than in the left arm. In contrast, heart rate was not different when the comparison was made between dominant and non-dominant exercises. This, however, might not apply to the present study, which was performed with dynamic voluntary and passive movement stimulation during the transition from rest to exercise. Additionally, Williamson et al. (35) found in humans that voluntary cycling exercise selectively activated the left-insular cortex, whereas passive cycling did not, suggesting that the left insular cortex may serve as a site for cortical regulation of cardiac vagal activity.

The results of the present study indicate that changes in R-R interval at the onset of passive exercise were not attenuated in the dominant forearm of racket players. These results indicate that cardiac vagal withdrawal induced by muscle mechanoreflex stimulation is well-maintained despite the long-term exposure to training. Finally, if there is any effect of racket sport training on the muscle mechanoreflex, it could be related to the increase in mechanoreceptor sensitivity in the non-dominant forearm due to "muscle disuse".

\section{Acknowledgments}

The authors appreciate the time and effort expended by all volunteer subjects in this study. We would also like to thank Dr. Rachel Drew for English grammar and style reviewing of the submitted version of the manuscript. 


\section{References}

1. McMahon SE, McWilliam PN. Changes in R-R interval at the start of muscle contraction in the decerebrate cat. $J$ Physiol 1992; 447: 549-562.

2. Araújo CGS, Nóbrega $A C L$, Castro CLB. Heart rate responses to deep breathing and 4-seconds of exercise before and after pharmacological blockade with atropine and propranolol. Clin Auton Res 1992; 2: 35-40.

3. Fagraeus L, Linnarsson D. Autonomic origin of heart rate fluctuations at the onset of muscular exercise. J Appl Physiol 1976; 40: 679-682.

4. Williamson JW, Fadel PJ, Mitchell JH. New insights into central cardiovascular control during exercise in humans: a central command update. Exp Physiol 2006; 91: 51-58.

5. Kaufman MP, Hayes SG, Adreani CM, Pickar JG. Discharge properties of group III and IV muscle afferents. Adv Exp Med Biol 2002; 508: 25-32.

6. Coote JH, Hilton SM, Perez-Gonzalez JF. The reflex nature of the pressor response to muscular exercise. J Physiol 1971; 215: 789-804.

7. al-Ani M, Munir SM, White MJ, Townend J, Coote JH. Changes in R-R variability before and after endurance training measured by power spectral analysis and by the effect of isometric muscle contraction. Eur J Appl Physiol Occup Physiol 1996; 74: 397-403.

8. Carrington CA, Fisher W, White MJ. The effects of athletic training and muscle contractile character on the pressor response to isometric exercise of the human triceps surae. Eur J Appl Physiol Occup Physiol 1999; 80: 337-343.

9. Fisher WJ, White MJ. Training-induced adaptations in the central command and peripheral reflex components of the pressor response to isometric exercise of the human triceps surae. J Physiol 1999; 520 (Pt 2): 621-628.

10. Hotta N, Yamamoto K, Sato K, Katayama K, Fukuoka Y, Ishida $\mathrm{K}$. Ventilatory and circulatory responses at the onset of dominant and non-dominant limb exercise. Eur J Appl Physiol 2007; 101: 347-358.

11. Miyamura M, Ishida K, Hashimoto I, Yuza N. Ventilatory response at the onset of voluntary exercise and passive movement in endurance runners. Eur J Appl Physiol Occup Physiol 1997; 76: 221-229.

12. Sato $\mathrm{K}$, Matsuo $\mathrm{H}$, Katayama K, Ishida K, Honda $\mathrm{Y}$, Katsumata $\mathrm{K}$, et al. Ventilatory and circulatory responses at the onset of voluntary exercise and passive movement in sprinters. Eur J Appl Physiol 2004; 92: 196-203.

13. Middlekauff HR, Chiu J, Hamilton MA, Fonarow GC, Maclellan WR, Hage A, et al. Muscle mechanoreceptor sensitivity in heart failure. Am J Physiol Heart Circ Physiol 2004; 287: H1937-H1943.

14. Nóbrega ACL, Araújo CGS. Heart rate transient at the onset of active and passive dynamic exercise. Med Sci Sports Exerc 1993; 25: 37-41.

15. Nóbrega ACL, Williamson JW, Friedman DB, Araújo CGS, Mitchell JH. Cardiovascular responses to active and passive cycling movements. Med Sci Sports Exerc 1994; 26 : 709-714.

16. Kaufman MP, Hayes SG. The exercise pressor reflex. Clin Auton Res 2002; 12: 429-439.

17. Hollander AP, Bouman LN. Cardiac acceleration in man elicited by a muscle-heart reflex. J Appl Physiol 1975; 38: 272-278.

18. Freyschuss $U$. Cardiovascular adjustment to somatomotor activation. The elicitation of increments in heart rate, aortic pressure and venomotor tone with the initiation of muscle contraction. Acta Physiol Scand Suppl 1970; 342: 1-63.

19. al-Ani M, Powell L, West J, Townend J, Coote JH. Exercise and diving, two conflicting stimuli influencing cardiac vagal tone in man. J Physiol 1995; 489 (Part 2): 603-612.

20. Farmer MR, Ross HF, Chowdhary S, Osman F, Townend $\mathrm{JN}$, Coote JH. GABAergic mechanisms involved in the vagally mediated heart rate response to muscle contraction as revealed by studies with benzodiazepines. Clin Auton Res 2003; 13: 45-50.

21. Saito $M$, Watanabe $H$, Mano $T$. Comparison of muscle sympathetic nerve activity during exercise in dominant and nondominant forearm. Eur J Appl Physiol Occup Physiol 1993; 66: 108-115.

22. Vianna LC, Oliveira RB, Araújo CGS. Age-related decline in handgrip strength differs according to gender. J Strength Cond Res 2007; 21: 1310-1314.

23. Norton K, Olds T. Anthropometrica. Marrickville: Southwood Press, 1996.

24. Rhea MR. Determining the magnitude of treatment effects in strength training research through the use of the effect size. J Strength Cond Res 2004; 18: 918-920.

25. Sinoway LI, Shenberger J, Leaman G, Zelis R, Gray K, Baily $\mathrm{R}$, et al. Forearm training attenuates sympathetic responses to prolonged rhythmic forearm exercise. J Appl Physiol 1996; 81: 1778-1784.

26. Sinoway LI, Hill JM, Pickar JG, Kaufman MP. Effects of contraction and lactic acid on the discharge of group III muscle afferents in cats. J Neurophysiol 1993; 69: 10531059.

27. Fisher JP, White MJ. Muscle afferent contributions to the cardiovascular response to isometric exercise. Exp Physiol 2004; 89: 639-646.

28. Haapasalo $H$, Kontulainen $S$, Sievanen $H$, Kannus $P$, Jarvinen M, Vuori I. Exercise-induced bone gain is due to enlargement in bone size without a change in volumetric bone density: a peripheral quantitative computed tomography study of the upper arms of male tennis players. Bone 2000; 27: 351-357.

29. Haapasalo $H$, Sievanen $H$, Kannus $P$, Heinonen A, Oja P, Vuori I. Dimensions and estimated mechanical characteristics of the humerus after long-term tennis loading. $J$ Bone Miner Res 1996; 11: 864-872.

30. Hayashi N, Koba S, Yoshida T. Disuse atrophy increases the muscle mechanoreflex in rats. J Appl Physiol 2005; 99: 1442-1445.

31. Rowell LB. Human cardiovascular control. Oxford: Oxford University Press; 1993.

32. Hachiya T, Blaber AP, Aizawa S, Saito M. Heart rate responses at onset of contraction. Int J Sports Med 2008; 29: 646-651.

33. Sinoway LI, Musch TI, Minotti JR, Zelis R. Enhanced maximal metabolic vasodilatation in the dominant forearms of tennis players. J Appl Physiol 1986; 61: 673-678. 
34. Gladwell VF, Fletcher J, Patel N, Elvidge LJ, Lloyd D, Chowdhary $\mathrm{S}$, et al. The influence of small fibre muscle mechanoreceptors on the cardiac vagus in humans. $J$ Physiol 2005; 567: 713-721.

35. Williamson JW, Nóbrega ACL, McColl R, Mathews D, Winchester $\mathrm{P}$, Friberg $\mathrm{L}$, et al. Activation of the insular cortex during dynamic exercise in humans. J Physiol 1997; 503 (Part 2): 277-283.

36. Drew RC, Bell MP, White MJ. Modulation of spontaneous baroreflex control of heart rate and indexes of vagal tone by passive calf muscle stretch during graded metaboreflex activation in humans. J Appl Physiol 2008; 104: 716-723.

37. Bell MP, White MJ. Cardiovascular responses to external compression of human calf muscle vary during graded metaboreflex stimulation. Exp Physiol 2005; 90: 383-391.

38. Saito M. Exercise-induced sympathetic activation is correlated with cerebral hemisphere laterality, but not handedness. Acta Physiol Scand 2000; 170: 111-118. 